\title{
Wilkie's Syndrome:A Rare Cause Of Duodenal Obstruction
}

\author{
Dr Badareesh L; Dr Siddharth Bhandary \\ Kasturba Medical College Manipal India \\ (Correspondence to Badareesh Lat drbadareesh.l@gmail.com)
}

\begin{abstract}
:
Introduction: Wilkie's syndrome is a rare disorder causing obstruction to the third part of duodenum due to acute angulation of superior mesenteric artery. It's a rare but surgically curable cause of duodenal obstruction. Case report: We present a case of $19 y e a r$ old male patient with chronic pain abdomen and vomiting which was correlated to obstruction of duodenum due to acute angulation of superior mesenteric artery on CECT abdomen. Patient's symptoms were dramatically improved following duodenojejunostomy. Conclusion: Wilkie's syndrome is more prevalent and underdiagnosed. Surgery is the treatment of choice.
\end{abstract}

Key words: Wilkie's syndrome, duodenojejunostomy

\section{Introduction:}

Superior mesenteric artery (SMA) syndrome is a very rare, life-threatening gastro-vascular disorder characterized by a compression of the third portion of the duodenum by the abdominal aorta (AA) and the overlying superior mesenteric artery. The syndrome is typically caused by an angle of $6^{\circ}-25^{\circ}$ between the AA and the SMA, in comparison to the normal range of $38^{\circ}-56^{\circ}$, due to a lack of retroperitoneal and visceral fat. In addition, the aortomesenteric distance is $2-8$ millimetres, as opposed to the typical 10-201. Clinically it presents with early satiety, nausea, bilious vomiting of large quantities of partially digested food, extreme "stabbing" postprandial abdominal pain (due to both the duodenal compression and the compensatory reversed peristalsis), abdominal distension/distortion, eructation, external hypersensitivity or tenderness of the abdominal area, and severe malnutrition accompanying spontaneous wasting ${ }^{2}$.

\section{Case report:}

A 19 year old male non-alcoholic, non-smoker patient visited with a history of pain abdomen since past 2 years. Pain was intermittent colicky in nature exacerbated by meals and associated with bile stained vomiting. Patient had history of loss weight about $20-30 \%$ of previous weight and felt lethargic. He underwent open appendicectomy at local hospital for such complaints about 1.5 years ago. But symptoms persisted and were exacerbated 2 weeks prior to hospitalisation.

On examination, he was moderately built poorly nourished young man with stable vital signs. Systemic examination remained unremarkable. An upper GI contrast study was ordered which is depicted in figure 1 and showed obstruction at third part of duodenum with proximal dilatation. To know the exact cause of the obstruction a CECT abdomen was taken. CT is shown in figure 2. It showed an acute angulation of superior mesenteric artery with the aorta (170) and a reduced aorta to SMA distance (6mm).

A working diagnosis of Wilkie's syndrome was then made. A trial of conservative treatment tried for about fifteen days without much improvement. Hence the patient was taken up for surgery. A retrocolic duodenojejunostomy was made. Following surgery patient had an uneventful post op recovery and was discharged on day 7. He was followed up for one year and remained asymptomatic and had gained weight adequately. 


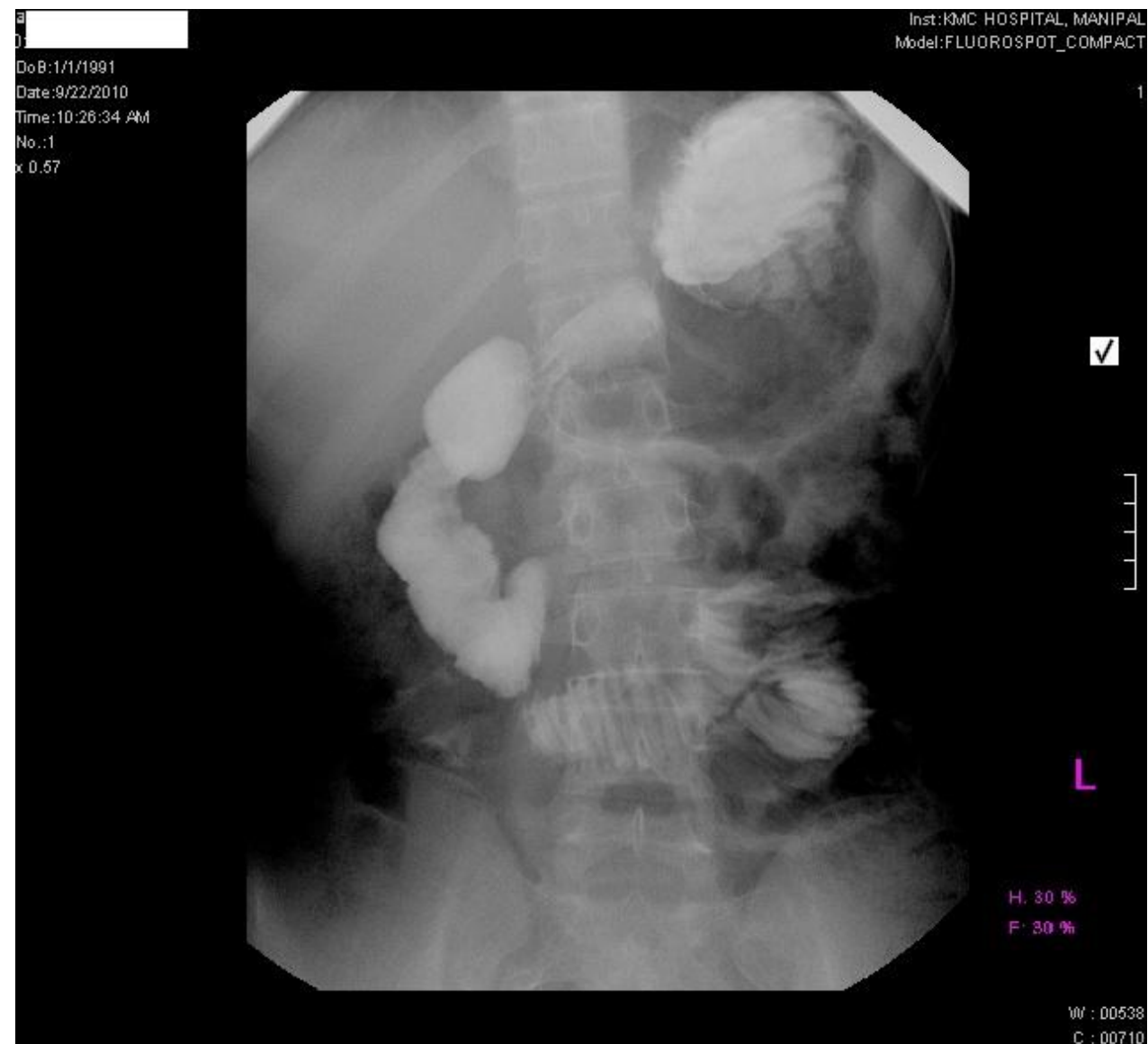

Figure 1- BMFT showing 3rd part duodenal obstruction

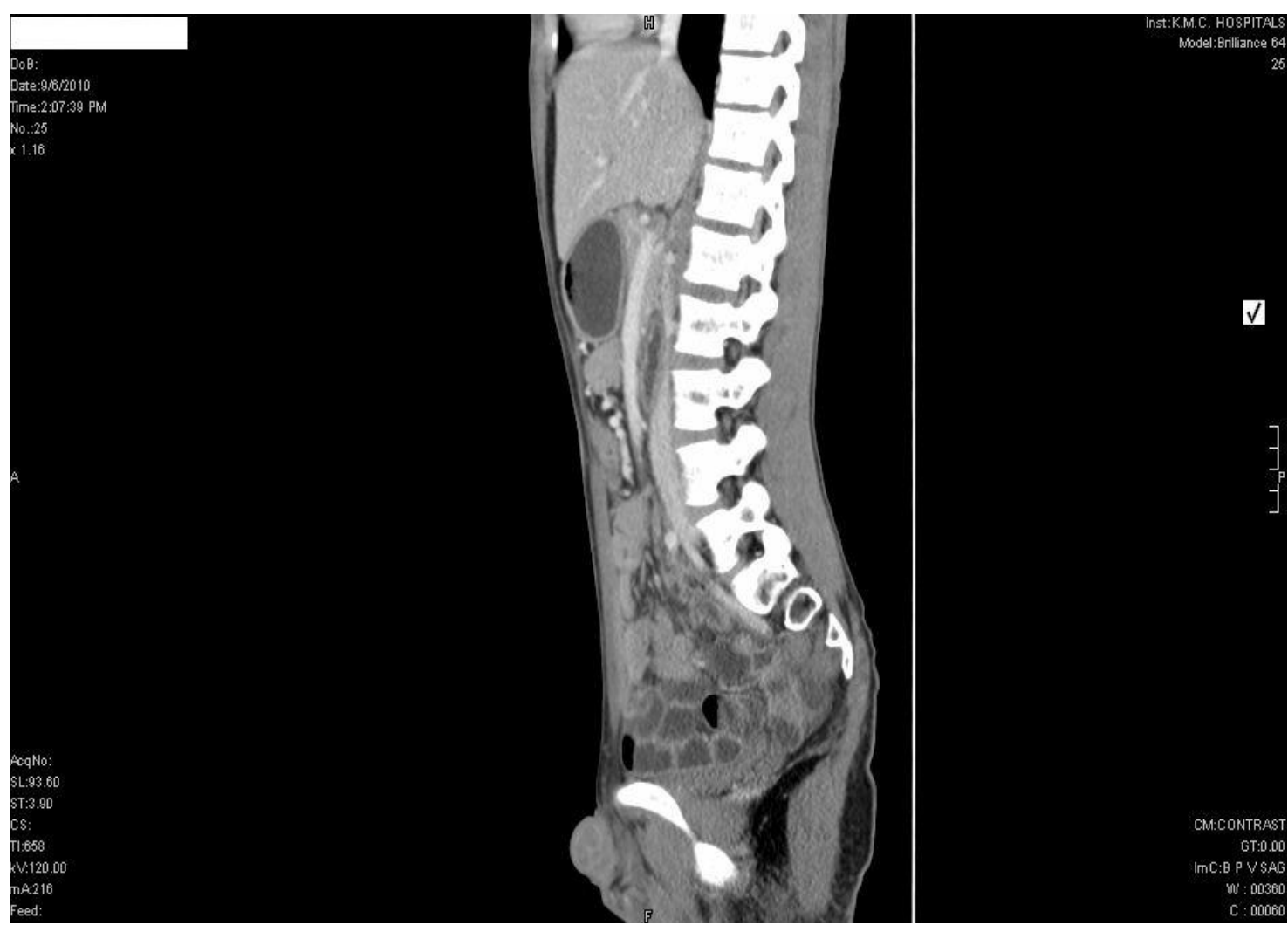

Figure 2- coronal CT abdomen showing duodenal compression by SMA 


\section{Discussion:}

Superior mesenteric artery (SMA) syndrome was described for the first time by Rokitansky in 1861 and has an incidence of $0.013-0.3 \%$ in the general population with a mortality rate of $33 \%{ }^{3}$. SMA syndrome is also known as Wilkie's syndrome, cast syndrome, mesenteric root syndrome, chronic duodenal ileus and intermittent arterio-mesenteric occlusion ${ }^{4}$. It occurs more frequently in women and clinically presents with vomiting, nausea, postprandial abdominal distension, pain, and weight $\operatorname{loss}^{5}$. The diagnosis is usually established by excluding other differential diagnoses ${ }^{6}$. Santer et al. and Applegate et al. were one of the first researchers to introduce CT findings of SMAS ${ }^{6}$. In their cases, they demonstrated gastric and duodenal dilatation, and the reduction of the distance between the SMA and the aorta ${ }^{6}$. Normally, fat and lymphatic tissues around the SMA provide protection to the duodenum against compression. Under conditions of severe weight loss, this cushion around the SMA is diminished, causing angulation and reduction in the distance between the aorta and the superior mesenteric artery. Normally, the aortomesenteric angle and aortomesenteric distance is $25^{\circ}$ to $60^{\circ}$ and 10 to $28 \mathrm{~mm}$, respectively. In SMA syndrome, both parameters are reduced, with values of $6^{\circ}$ to $15^{\circ}$ and 2 to $8 \mathrm{~mm}^{7}$.

Traditionally, treatment has consisted of conservative measures such as nasogastric decompression and hyperalimentation followed by oral feeding with frequent small meals. Posturing maneuvers during meals and motility agents may be helpful in some patients. Surgery may be considered if conservative treatment fails ${ }^{8}$. The first proposed surgery for SMAS was described as duodenojejunostomy by Bloodgood ${ }^{9}$. Duodenojejunostomy is effective in the majority of patients ${ }^{8}$. In this procedure compressed portion of the duodenum is released and an anastomosis is created between the duodenum and jejunum anterior to the superior mesenteric artery.

\section{Conclusion:}

Based on our experience, we feel that Wilkie's syndrome is far more prevalent and is underdiagnosed. Hence we strongly recommend that patients with chronic vague upper abdominal symptoms need to be screened with CECT abdomen to rule out this disease.

\section{References:}

[1]. Avinash Shetty (2006-07-16). "Superior Mesenteric Artery Syndrome". eMedicine.WebMD. Retrieved 2008-04-09.

[2]. Baltazar U, Dunn J, Floresguerra C, Schmidt L, et al. (2000). "Superior mesenteric artery syndrome: an uncommon cause of intestinal obstruction". South. Med. J. 93 (6): 606-8.PMID 10881780.

[3]. Rokitansky, Handbuch der pathologischen Anatomie, vol. 3 Braunmüller \& Seidel,Wien, Austria, 1 st edition, 1842.

[4]. Laffont I, Bensmail D, Rech C, et al. (2002). "Late superior mesenteric artery syndrome in paraplegia: case report and review". Spinal Cord 40 (2): 88-91

[5]. P. Ylinen, J. Kinnunen, and K. Hockerstedt, "Superior mesenteric artery syndrome. A follow-up study of 16 operated patients," Journal of Clinical Gastroenterology, vol. 11, no. 4, pp. 386-391, 1989.

[6]. Birsen Ünal, Aykut Aktaş, Gökhan Kemal, et al. Superior mesenteric artery syndrome: CT and ultrasonography findings Diagn Interv Radiol 2005; 11:90-95

[7]. Lippl F, Hannig C, Weiss W, et al. Superior mesenteric artery syndrome: diagnosis and treatment from the gastroenterologist's view. J Gastroenterol.2002;37:640-3.

[8]. Hines JR, Gore RM, Ballantyne GH. Superior mesenteric artery syndrome. Diagnostic criteria and therapeutic approaches. Am $J$ Surg. 1984;148:630-2. [PubMed]

[9]. Rikki Singal, Pradeep Kumar Sahu, Mukesh Goel et al Superior mesenteric artery syndrome: A case report. North American Journal of Medical Sciences 2010 August, Volume 2. No. 8.(392-94). 